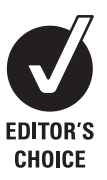

See Editorial, p 350

${ }^{1}$ Falls and Syncope Service, and Institute for Ageing and Health, Newcastle General Hospital, Newcastle upon Tyne, UK; ${ }^{2}$ Institute of Health and Society, University of Newcastle upon Tyne, Newcastle upon Tyne, UK; ${ }^{3}$ Department of Cardiology, Freeman Hospital, Newcastle upon Tyne, UK; ${ }^{4}$ Trinity College, College Green, Dublin, Ireland

Correspondence to:

Dr S W Parry, Falls and Syncope Service, Royal Victoria Infirmary, Queen Victoria Road, Newcastle upon Tyne NE1 4LP, UK swparry@hotmail.com

Accepted 25 November 2008 Published Online First

5 January 2009

\title{
Pacing in elderly recurrent fallers with carotid sinus hypersensitivity: a randomised, double-blind, placebo controlled crossover trial
}

\author{
S W Parry, ${ }^{1}$ N Steen, ${ }^{2}$ R S Bexton, ${ }^{3}$ M Tynan, ${ }^{3}$ R A Kenny ${ }^{4}$
}

\section{ABSTRACT}

Objectives: While carotid sinus syndrome (CSS) is traditionally defined by the association of carotid sinus hypersensitivity (CSH) with syncope, uncertainty remains over the role, if any, of complex pacing in patients with CSH and unexplained or recurrent falls. We sought to clarify the role of dual chamber pacing in this patient group in the first placebo-controlled study in CSH.

Design: Randomised, double-blind, crossover, placebocontrolled trial.

Setting: Specialist falls and syncope facility.

Patients: Consecutive subjects aged over 55 years with $\mathrm{CSH}$ as the sole attributable cause of three or more unexplained falls in the 6 months preceding enrolment.

Intervention: Dual-chamber permanent pacing with ratedrop response programming. The pacemaker was switched on (DDD/RDR) or off (ODO (placebo)) for 6 months, then crossed over to the alternate mode for a further 6 months, in randomised, double-blind fashion.

Main outcome measure: The primary outcome measure was number of falls in paced and non-paced modes.

Results: Twenty-five of 34 subjects (mean 76.8 years (SD 9.0), 27 (79\%) female) recruited completed the study. Pacing intervention had no effect on number of falls (4.04 (9.54) in DDD/RDR mode, 3.48 (7.22) in OD0; relative risk of falling in $\mathrm{ODO}$ mode $0.82,95 \% \mathrm{Cl} 0.62$ to 1.10).

Conclusion: Permanent pacing intervention had no effect on fall rates in older patients with CSH. Further work is urgently needed to clarify the role, if any, of complex pacing in this patient group.

Carotid sinus hypersensitivity (CSH) has been causally implicated in spontaneous syncope and presyncope and dizziness for more than 70 years, ${ }^{12}$ but it is only in the last decade or so that increasing interest has focused on the association between $\mathrm{CSH}$ and non-accidental or unexplained falls in the absence of overt loss of consciousness. ${ }^{3-9}$ Recent guidelines ${ }^{10-12}$ have favoured permanent pacing as the treatment of choice in the cardioinhibitory and mixed subtypes of CSH associated with syncope, but there is controversy over the role of pacing in patients with $\mathrm{CSH}$ and non-accidental falls. ${ }^{13}$ The largest randomised study to date investigating the role of pacing in this patient population showed a two-thirds reduction in the number of falls in the intervention group, ${ }^{8}$ but the study is hampered by the absence of blinding and a placebo arm, both important components in intervention studies in neurally mediated discorders. ${ }^{14}$ To address these questions, we investigated whether cardiac pacing reduced events in patients with recurrent falls and cardioinhibitory carotid sinus hypersensitivity in an exploratory, randomised, double-blind, crossover, placebo-controlled trial.

\section{METHODS \\ Subjects}

Consecutive subjects aged 55 years and over presenting to the emergency department or the syncope service with three or more unexplained falls (per self report and witness account where available) and no overt syncope in the preceding 6 months were investigated according to national and international guidelines. ${ }^{9-12}{ }^{15}$ Unexplained falls were defined as those for which no immediate cause was apparent, either intrinsic (eg, stroke, myocardial infarction [MI], gastro-intestinal haemorrhage or other medical diagnosis) or extrinsic (eg, simple trip over carpet, ill-fitting footwear) following detailed clinical evaluation per national and international guidance. ${ }^{915}$ Syncope work-up included morning active stand to evaluate orthostatic hypotension, surface electrocardiogram (ECG), head-up tilt table testing, ambulatory ECG and blood pressure monitoring, echocardiogram and carotid sinus massage (CSM) ${ }^{10-12}$ Those with an otherwise negative work-up and cardioinhibitory (CI) or mixed ( $\mathrm{Mx}$ ) subtypes ( $>3 \mathrm{~s}$ asystole induced by $5 \mathrm{~s}$ of bilateral sequential CSM performed supine and erect ${ }^{16}$ with $(\mathrm{Mx})$ or without (CI) a $50 \mathrm{~mm} \mathrm{Hg}$ fall in systolic blood pressure following $600 \mu \mathrm{g}$ injection of intravenous atropine) of $\mathrm{CSH}$ as the sole attributable cause of their falls were invited to participate in the pacing intervention study.

\section{Exclusions}

Subjects were excluded if there were other possible causes of their falls or if they had moderate to severe cognitive impairment (Mini Mental State Examination $^{17} \leqslant 15 / 30$ ), stroke or MI within 3 months (or other contraindication to $\mathrm{CSM}^{10}{ }^{12}$ ) or any history of syncope.

The study had approval from the local Research Ethics Committee.

\section{Intervention}

All subjects underwent dual-chamber permanent pacemaker implantation. In all subjects, the device implanted was the Kappa DR (Medtronic, Minneapolis, Minnesota) system programmed to the rate-drop response ( $R D R$ ) algorithm, which allows backup pacing for patients with occasional drops in heart rate..$^{18}$ Following pacemaker implantation, all 
subjects' pacemakers were programmed to on (ie, in DDD/RDR mode) for a 1-month run-in period in order to ensure that they were unaware of pacing intervention. At 1 month, subjects were randomised (by table of random numbers) in double-blind fashion to either continue in DDD/RDR mode, or for the pacing to be turned off (ODO mode). Six months later, patients crossed over to the opposite mode for the remaining 6 months of the study.

Permanent crossover to the DDD/RDR mode occurred if a further indication for pacing therapy was diagnosed, if syncope supervened or at the behest of other physicians caring for the patient concerned.

\section{Event monitoring}

All subjects completed daily fall diaries returned at weekly intervals using prepaid postage to avoid confounding due to inaccurate recall of falls. Information from the falls diaries was held at a central database; failure of diary return initiated a telephone prompt to keep diary returns contemporaneous.

\section{Outcome measures}

The primary outcome measure was the number of falls. This was assessed in two ways: whether or not a participant falls at all during each study period and the number of falls in each study period. The secondary outcome measure was the time to first fall.

\section{Sample size}

Previous work (based on preliminary data ${ }^{5}$ ) suggested that we might expect pacing to result in a reduction in falling of around $27 \%$ and that this corresponded to an effect size of approximately 0.5 . This was a significantly lower treatment effect than the $66 \%$ reduction in fall rates seen in the SAFE-PACE study, ${ }^{8}$ but these data were not available at the time of power calculation. Based on the $27 \%$ treatment effect, a sample size calculation for a paired comparison indicated that a total sample of 32 subjects would be required to detect an effect size of 0.5 with $80 \%$ power assuming a type 1 error rate of $5 \%$. A target sample size of 34 was specified to allow for drop out during the study (an attrition rate of $5.9 \%$ ).

\section{Statistical analysis}

McNemar test was used to compare the number of participants falling during the 6 months with their pacemaker turned off with the number of participants falling during the 6 months with their pacemaker turned on. The numbers of falls in the two periods were compared using negative binomial regression with variation between subjects and variation between periods included as fixed effects and the log of the number of days at risk included as an offset. The time to first fall was investigated using Kaplan-Meier plots and survival analysis using SPSS Version 10.

\section{RESULTS}

\section{Baseline clinical characteristics}

Thirty-four patients were recruited to the study. Baseline clinical characteristics of the study population, including age, sex, MMSE, falls history and consequences, medications and comorbidity, are shown in table 1. Haemodynamic consequences of baseline carotid sinus massage are shown in table 2.

\section{Falls-outcome data}

Twenty-five patients completed the study. Three subjects permanently crossed-over to DDD/RDR pacing from ODO mode (one because of syncope, two because of physician preference), one subject refused further participation because of pacemaker erosion, four subjects died (three from ischaemic heart disease, one postemergency colectomy for ischaemic bowel), and one suffered a total anterior circulation stroke.

Ninety-one point four per cent of falls diaries were completed and returned for analysis. None of the subjects reported awareness of pacing during the 1-month prerandomisation period, during which they experienced a mean of 0.64 falls (SD 1.07). The number of falls for each participant is described in table 3. There were a mean of 3.48 (7.22) falls during the 6 months in ODO mode, compared with 4.04 (9.54) falls during the 6 months in DDD/RDR mode. On average, there were 0.56 fewer falls per participant during the period with the pacemaker turned on. Analysing the number of falls using negative binomial regression indicates that the relative risk of falling with the pacemaker turned on compared with the pacemaker turned off is 0.82 with a $95 \%$ CI of 0.62 to 1.10 .

Of the 25 participants completing the study, seven did not fall in either period, and 12 fell in both periods. Of the remaining six participants, two fell only while their pacemaker was turned off, and four fell only while their pacemaker was turned on. This difference was not significant ( $p=0.69$, McNemar test).

Survival analysis (treating the pacemaker on/off groups as independent samples) showed that there were no significant differences in time to first fall between DDD/RDR and ODO modes (fig $1 ; \mathrm{p}=0.57$ ).

\section{Pacemaker interrogation following falls}

On three occasions, subjects presented to the pacing department within $4 \mathrm{~h}$ of falling. Unfortunately, on these occasions, the pacemakers were in ODO mode, so bradyarrhythmias could not be detected. There was no evidence of malignant tachydysrrhythmia in any of these cases, however. Two of the three patients who died suddenly at home (coroner's postmortem reported ischaemic heart disease as the cause of death in each case) were in ODO mode at the time of death. Although the pacemakers were recovered and interrogated, there were no diagnostically useful data retrieved.

\section{Consequences of falls}

There were relatively few injuries during the study period. During the DDD/RDR study period, one subject sustained a wrist fracture, another suffered a scalp laceration requiring sutures, and a third required medical attention for a sprained ankle. During the ODO study period, one subject suffered a back injury and severe bruising secondary to falls, while the patient who suffered the wrist fracture sought medical advice for falls on three different occasions.

\section{DISCUSSION}

Our study suggests that the benefits of permanent pacing in subjects with unexplained falls and CSH in this, the first, randomised, double-blind, placebo-controlled trial in its field, are at best modest. There was no evidence that pacing intervention reduced the percentage of patients who fell. The rationale for pacing in such individuals is either that they are experiencing transient "micro-syncope" with collapse and memory of a fall rather than loss of consciousness or that the sudden catastrophic cerebral hypoperfusion caused by cardiac asystole and hypotension secondary to CSH causes transient balance abnormalities resulting in falls. As both falls and syncope are multifactorial in aetiology in many older patients, 
Table 1 Baseline clinical characteristics of subjects with carotid sinus hypersensitivity and unexplained falls

\begin{tabular}{|c|c|}
\hline & Patients with unexplained falls $(n=34)$ \\
\hline Age, years (SD) & 76.8 (9) (range 56 to 89 ), median 77.5 \\
\hline \multicolumn{2}{|l|}{ Sex } \\
\hline Female & $27(79 \%)$ \\
\hline Male & $7(21 \%)$ \\
\hline Mean Mini-Mental State Examination (SD) (median) & $27(2.5)(27)$ \\
\hline Mean no falls in 6 months & 7 (median 6) (range 3 to 40 ) \\
\hline Mean symptom duration & 13 months (median 9) (range 5 to 60 ) \\
\hline Mean no episodes ever (SD) & 13 (12.8) (median 8) (range 3 to 50 ) \\
\hline Hospital admissions/A\&E attendances* & $16(47 \%)$ \\
\hline Fractures* & $8(24 \%)$ \\
\hline Soft-tissue injuries* & $14(41 \%)$ \\
\hline Requiring medical attention & $9(26 \%)$ \\
\hline Facial bruising & $5(15 \%)$ \\
\hline \multicolumn{2}{|l|}{ Medications } \\
\hline Polypharmacy & $7(20 \%)$ \\
\hline Cardiovascular & $11(32 \%)$ \\
\hline Psychoactive & $4(12 \%)$ \\
\hline None & $6(18 \%)$ \\
\hline Ischaemic heart disease & $8(24 \%)$ \\
\hline Hypertension & $5(15 \%)$ \\
\hline Osteoarthritis & $9(26 \%)$ \\
\hline Stroke & $3(9 \%)$ \\
\hline Dementia & $3(9 \%)$ \\
\hline Chronic obstructive pulmonary disease & $8(24 \%)$ \\
\hline Type II diabetes & $3(9 \%)$ \\
\hline
\end{tabular}

*Secondary to falls/syncope; polypharmacy: four or more prescribed drugs.

we were careful to exclude other causes of these problems in a systematic and evidence-based manner. Our study population was thus a relatively "clean" one, although the high preenrolment fall burden was striking, suggesting the possibility of undetected falls risks.

The quality of the falls outcome data is excellent, with a $91.4 \%$ fall diary completion rate, but while the current study benefits from a more powerful study design (placebo-controlled with crossover) than that of Kenny et $a l^{8}$ (no placebo limb) or Crilley et $a l^{4}$ (retrospective, with poorly defined symptom complexes) studies, it suffers from the small number of patients completing the study. Though the baseline sample met the initial power calculation requirements, the $26 \%$ attrition rate (vs an expected 5\%) left the study with fewer patients than anticipated. As a result, the study may be significantly

Table 2 Results of carotid sinus massage in subjects with carotid sinus hypersensitivity and unexplained falls

\begin{tabular}{ll}
\hline & Patients with unexplained falls ( $\mathbf{n}=\mathbf{3 4})$ \\
\hline CSH subtype & $\mathrm{Cl} 15(44 \%)$ \\
& $\mathrm{Mx} 17(50 \%)$ \\
& $\mathrm{U} 2(6 \%)$ \\
Mean max asystole (S) (SD) & $5.06(1.5)$ \\
Mean max VD (mm Hg) (SD) & $76.7(35.2)$ \\
VD postatropine ( $=19)$ & \\
$\quad$ Mean VD supine (mm Hg) (SD) & $73.4(15.1)^{*}$ \\
Mean VD upright (mm Hg) (SD) & $87.2(18.6)$ \\
Laterality of initial positive CSM & $24(71 \%)$ right \\
Initial CSM positive upright & $20(59 \%)$ \\
LOC during CSM & $22(64 \%)$ \\
Amnesia for LOC & $21(95 \%$ of LOC) 12 (57\%) upright \\
\hline
\end{tabular}

${ }^{*} \mathrm{p}=0.000034$

$\mathrm{Cl}$, cardioinhibitory; $\mathrm{CSH}$, carotid sinus hypersensitivity; CSM, carotid sinus massage; LOC, loss of consciousness; max, maximum; Mx, mixed; U, unclassifiable; VD, vasodepressor response. underpowered, although the lower interval for relative risk of falling (0.62; corresponding to the most optimistic estimate of the clinical effectiveness in the SAFE-PACE study) implies that the impact of pacing is not as large as the effects observed in non-blinded studies, ${ }^{8}$ lessening the impact of the lower than anticipated study completion rate.

Carotid sinus massage induced cardioinhibitory and mixed subtypes in almost equal proportions. There was a profound fall in mean systolic blood pressure during CSM $(76.2 \mathrm{~mm} \mathrm{Hg}$; table 2) in the group overall, with a similar vasodepressor response evident in those undergoing massage post-atropine (table 2). Although a clinical commonplace, the significant increase in vasodepression in the head-up tilt position during CSM has not previously been documented under experimental conditions. While none of the patients in this study experienced neurological (or any other) complications due to CSM, the larger degree of vasodepression during CSM in the upright position may predispose towards watershed-type cerebrovascular events. ${ }^{19}$ Though a causal association between profound vasodepression and neurological complications during CSM has not been formally established, the current pragmatic approach of performing massage supine initially and to ensure a long period of supine recovery before ambulation post-CSM related vasodepression is reinforced by these findings. ${ }^{10}$ Similarly, the fact that $71 \%$ of initially positive CSM episodes were on the right side reinforces the suggestion that massage be performed sequentially right- then left-sided. ${ }^{10}$ The high proportion of subjects whose initial positive response to CSM was in the upright position (59\%) is noteworthy and reinforces the importance of repeating negative supine CSM in the upright position. ${ }^{16}$

The high death rate in this study is worthy of mention. Three died suddenly at home, with coroner's post-mortem results showing only ischaemic heart disease. None of these subjects had suffered a fatal myocardial infarction, raising the spectre of 
Table 3 Number of days at risk and number of falls for each subject by period

\begin{tabular}{|c|c|c|c|c|c|c|}
\hline \multicolumn{5}{|l|}{ No of falls } & \multicolumn{2}{|l|}{ No of days } \\
\hline $\begin{array}{l}\text { Running in } \\
\text { period }\end{array}$ & $\begin{array}{l}\text { Pacemaker } \\
\text { turned off* }\end{array}$ & $\begin{array}{l}\text { Pacemaker } \\
\text { turned on: }\end{array}$ & Total & Off-on & $\begin{array}{l}\text { Pacemaker } \\
\text { turned off* }\end{array}$ & $\begin{array}{l}\text { Pacemaker } \\
\text { turned on } \dagger\end{array}$ \\
\hline 0 & 0 & 0 & 0 & 0 & 182 & 196 \\
\hline 0 & 0 & 0 & 0 & 0 & 81 & 377 \\
\hline 0 & 0 & 0 & 0 & 0 & 189 & 175 \\
\hline 0 & 0 & 0 & 0 & 0 & 182 & 189 \\
\hline 0 & 0 & 0 & 0 & 0 & 182 & 186 \\
\hline 0 & 0 & 0 & 0 & 0 & 203 & 196 \\
\hline 0 & 0 & 0 & 0 & 0 & 182 & 210 \\
\hline 0 & 0 & 1 & 1 & -1 & 182 & 185 \\
\hline 0 & 0 & 1 & 1 & -1 & 186 & 196 \\
\hline 0 & 1 & 0 & 1 & 1 & 189 & 189 \\
\hline 0 & 0 & 2 & 2 & -2 & 144 & 230 \\
\hline 0 & 1 & 1 & 2 & 0 & 182 & 186 \\
\hline 0 & 2 & 0 & 2 & 2 & 188 & 169 \\
\hline 0 & 1 & 1 & 2 & 0 & 190 & 168 \\
\hline 0 & 0 & 2 & 2 & -2 & 170 & 183 \\
\hline 0 & 1 & 2 & 3 & -1 & 203 & 182 \\
\hline 0 & 2 & 1 & 3 & 1 & 182 & 185 \\
\hline 0 & 2 & 3 & 5 & -1 & 174 & 189 \\
\hline 2 & 3 & 1 & 6 & 2 & 182 & 190 \\
\hline 2 & 1 & 4 & 7 & -3 & 182 & 182 \\
\hline 3 & 9 & 3 & 15 & 6 & 200 & 199 \\
\hline 2 & 6 & 10 & 18 & -4 & 168 & 193 \\
\hline 3 & 5 & 14 & 22 & -9 & 161 & 176 \\
\hline 2 & 24 & 7 & 33 & 17 & 182 & 167 \\
\hline 2 & 43 & 34 & 79 & 9 & 168 & 182 \\
\hline \multicolumn{7}{|l|}{ Means: } \\
\hline 0.64 & 4.04 & 3.48 & 8.16 & $0.56 \%$ & 177.4 & 195.2 \\
\hline
\end{tabular}

occult ventricular tachyarrhythmias as a potential cause of symptoms and sudden death. Potentially causative ventricular tachycardia has been documented during electrophysiology studies in subjects with carotid sinus syncope. ${ }^{20}$ All three of the deceased had normal $24 \mathrm{~h}$ ambulatory ECG recordings but had features of ischaemic heart disease on surface ECG, but as with all the study patients, none had suffered syncope at any time and had a negative "syncope work-up" as detailed above, including normal ejection fractions on echocardiography. The

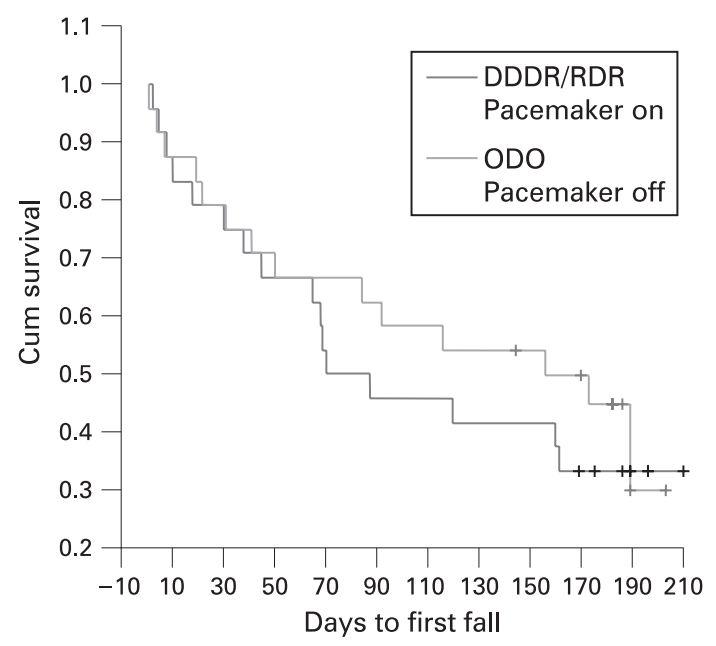

Figure 1 Time to first fall: survival analysis. presence of any putative malignant tachyarrhythmias must thus remain speculative.

The distribution of falls during the paced and non-paced periods hints at a placebo effect. In favour of this hypothesis is the overall reduction in the number of falls in both 6-month periods compared with the pre-enrolment fall burden (mean seven falls) and the high number of subjects who experienced no falls at all during the course of the study (regardless of pacing mode status). It is possible that the short duration of follow-up in paced and non-paced modes may be a source of bias, though this is unlikely given the high mean and median number of falls in the 6 months prior to enrolment. The highly symptomatic nature of the patients enrolled may also imply "regression to the mean" during follow-up rather than either a placebo effect.

The high proportion of subjects with loss of consciousness during CSM (64\%; table 2) is noteworthy. In the context of the investigation of unexplained syncope, recent guidelines suggest that CSM is diagnostic only in the presence of both haemodynamic changes and symptom reproduction, ${ }^{12}$ the socalled "method of symptoms." 21 Our patients clearly could not reproduce a fall during the confines of laboratory CSM, and so do not fit conventional diagnostic criteria. Our group has recently shown that up to one-third of community dwelling elders fulfil criteria for CSH during CSM regardless of symptoms of falls, dizziness or syncope, ${ }^{22}$ so the attribution of $\mathrm{CSH}$ in a causal role in our patients' falls must now be open to speculation.

In conclusion, we found no evidence that pacing helped improve the fall burden in patients with $\mathrm{CSH}$ who had 
undergone a comprehensive battery of investigations to exclude any other causes for their symptoms. The overall number of falls fell, but the number of falls was similar regardless of pacing or placebo intervention in this crossover study. Although enrolment initially fulfilled our sample estimate, our study population suffered a high attrition rate, leaving the study underpowered for the final analysis. This work is important in showing that a placebo-controlled pacing study is feasible and well tolerated in this population, and further work is needed to establish the continued relevance of permanent pacing in unexplained fallers with carotid sinus hypersensitivity.

Competing interests: None.

Ethics approval: Ethics approval was provided by the Newcastle Local Research Ethics Committee.

Patient consent: Obtained.

\section{REFERENCES}

1. Roskam J. Un syndrome noveau. Syncopes cardiaques gràves et syncopes répétées par hyperréflectivité sinocarotidienne. Presse Med 1930;38:590-1.

2. Weiss S, Baker JP. Carotid sinus reflex in health and disease: its role in the causation of fainting and convulsions. Medicine 1933;12:297-354.

3. McIntosh SJ, Lawson J, Kenny RA. Clinical characteristics of vasodepressor, cardioinhibitory, and mixed carotid sinus syndrome in the elderly. Am J Med 1993;95:203-8.

4. Crilley JG, Herd B, Khurana CS, et al. Permanent cardiac pacing in elderly patients with recurrent falls, dizziness and syncope, and a hypersensitive cardioinhibitory reflex. Postgrad Med J 1997;73:415-18.

5. Davies AJ, Kenny RA. Falls presenting to the accident and emergency department: types of presentation and risk factor profile. Age Ageing 1996;25:362-5.

6. Parry SW, Steen IN, Baptist M, et al. Amnesia for loss of consciousness in patients with carotid sinus syndrome: Implications for presentations with falls. J Am Coll Cardiol 2005;45:1840-3.
7. Parry SW, Baptist M, Kenny RA. Drop attacks in older adults: systematic assessment has high diagnostic yield. J Am Geriatr Soc 2005;53:74-8.

8. Kenny RA, Richardson DA, Steen N, et al. Carotid sinus syndrome: a modifiable risk factor for nonaccidental falls in older adults (SAFE PACE). J Am Coll Cardiol 2001;38:1491-6

9. National Institute for Health and Clinical Excellence. Falls: the assessment and prevention of falls in older people. London: HMSO, 2005.

10. Kenny RA, O'Shea D, Parry SW. The Newcastle protocols for head-up tilt table testing in the diagnosis of vasovagal syncope, carotid sinus hypersensitivity, and related disorders. Heart 2000;83:564-9.

11. Vardas PE, Auriccio A, Blanc JJ, et al. Guidelines for cardiac pacing and cardiac resynchronization therapy. The Task Force for cardiac pacing and cardiac resynchronization therapy of the European Society of Cardiology. Europace 2007; 9:959-98.

12. Brignole M, Alboni $\mathrm{P}$, Benditt DG, et al. Guidelines on management (diagnosis and treatment) of syncope. Eur Heart J 2001;22:1256-306.

13. McAnulty J. Carotid sinus massage in patients who fall: will it define the role of pacing? J Am Coll Cardiol 2001;38:1497.

14. Sheldon R, Rose S. Components of clinical trials for vasovagal syncope. Europace 2001;3:233-40.

15. American Geriatrics Society. Guideline for the prevention of falls in older persons. J Am Geriatr Soc 2001;49:664-72.

16. Parry SW, Richardson DA, Sen B, et al. Diagnosis of carotid sinus hypersensitivity in older adults: carotid sinus massage in the upright position is essential. Heart 2000;82:22-3.

17. Folstein MF, Folstein SE, McHugh PR. "Mini mental state." A practical method for grading the cognitive state of patients for the clinician. J Psychiatr Res 1975;12:189-98.

18. Gammage MD. Rate-drop response programming. Pacing Clin Electrophysiol 1997;20:841-3.

19. Davies AJ, Kenny RA. Frequency of neurologic complications following carotid sinus massage. Am J Cardiol 1998;8:1256-7.

20. Nelson SD, Kou WH, De Buitleir $M$, et al. Value of programmed ventricular stimulation in presumed carotid sinus syndrome. Am J Cardiol 1987;60:1073-7.

21. Brignole M, Menozzi C. Carotid sinus syndrome: diagnosis, natural history and treatment. Eur J Cardiac Pacing 1992;4:247-54.

22. Kerr SR, Pearce MS, Brayne C, et al. Carotid sinus hypersensitivity in asymptomatic older persons: implications for diagnosis of syncope and falls. Arch Intern Med 2006; 166:515-20. 